\title{
COVID-19 presenting like Steven Johnson Syndrome in a pediatric patient
}

\author{
Aslinur Ozkaya Parlakay ${ }^{1}$, Serhan Ozcan ${ }^{1}$, Betul Karaatmaca ${ }^{1}$, Mehmet Tiftik ${ }^{1}$, Güzin \\ $\mathrm{Cinel}^{2}$, and Emrah Senel ${ }^{1}$ \\ ${ }^{1}$ Ankara Children's Hematology Oncology Training and Research Hospital \\ ${ }^{2}$ Hacettepe University, İhsan Dogramacı Children's Hospital
}

June 22, 2020

\begin{abstract}
Since the outbreak of 2019 novel coronavirus infection (COVID-19) 5,698,246 cases were infected with COVID-19, there were 352,461 deaths. Various characteristics of COVID-19 were presented but to our knowledge this is the first pediatric case, an eight year old boy, presenting like Steven Johnson Syndrome. In patients with atypical presentations and pulmonary findings COVID-19 should be kept in mind.
\end{abstract}

Coronaviruses are large, enveloped, positive strand RNA viruses that can be divided into 4 genera: alpha, beta, delta, and gamma, of which alpha and beta CoVs are known to infect humans, which is called human coronaviruses (HcoVs). Emergence of $2019-\mathrm{nCoV}$ has attracted global attention, and WHO has declared the COVID-19 a public health emergency of international concern ${ }^{1}$. In the first retrospective study on the epidemiological characteristics and transmission dynamics of children's COVID-19 in China various characteristics were presented but to our knowledge this is the first pediatric case presenting like Steven Johnson Syndrome.

An eight-year-old boy who had been healthy previously other than rash history with amoxicilin clavulonate 2 times, when admitted with fever was prescribed amoxicillin-clavulonate for tonsillitis inadvertently, developped rash a day after, increasing in intensity, with respiratory symptomps (ronchuses at basal zones) diagnosed as Steven Johnson Syndrome under therapy of $2 \mathrm{mg} / \mathrm{kg}$ prednisolone and $0.5 \mathrm{~g} / \mathrm{kg}$ IVIG was admitted to intensive care unit.

On admission, he had respiratory distress, hypoxia, cough and bilateral pericardiac infiltration in chest Xray (CXR), cofluenting hyperemic rash all over the body especially trunk area(Figure 1). Clindamycin and oseltamivir was initiated. On day 2 of admission while rash was decreasing, respiratory failure increased, chest x-ray worsened, computed tomography showed intense consolidation, especially at basal posterior zones, ground glass opacities and emphysematous changes at upper zones which were not specific to previous COVID CTs (Fig 2). But still nasopharyngeal aspirate was studied for COVID which was negative. These changes were attributed to SJS lung involvement. So cyclosporin was initiated. Patient got better under therapy, rash and oxygen need dissappeared. But still chest $\mathrm{x}$ ray continued to worsen so deep tracheal aspirate was studied 2 days after the nasophrayngeal aspirate which was positive. Azitromycine, hydroxychloroquinine was added to therapy. Both respiratory symptoms and chest xray responded well.

As of May 27th, 2020, 5,698,246 cases were infected with COVID-19, there were 352,461 deaths. In a previous report out of the 1391 children assessed a total of 171 (12.3\%) were confirmed to have COVID-19 infection. The median age of the infected children was 6.7 years. Fever was present in $41.5 \%$ of the children at any 
time during the illness. Other common signs and symptoms included cough and pharyngeal erythema. A total of 27 patients $(15.8 \%)$ did not have any symptoms of infection or radiologic features of pneumonia. Whereas 12 patients had radiologic features of pneumonia but did not have any symptoms of infection ${ }^{2}$.

Children's COVID-19 cases were less severe than adults' cases, which may be related to both exposure and host factors ${ }^{1}$. It was speculated that children were less sensitive to COVID-19 because the maturity and function (e.g., binding ability) of ACE2 in children may be lower than that in adults ${ }^{3}$. Also, children often experience respiratory infections (e.g., respiratory syncytial virus (RSV)) in winter, and may have higher levels of antibody against virus than adults and children's immune system is still developing, and may respond to pathogens differently to adults.

Cylosporin might also be effective in treatment of COVID-19, as there are few studies regarding that it could be an option. In a previous study it was stated that cylosporin A inhibits the replication of diverse coronaviruses at non-cytotoxic, low-micromolar concentrations ${ }^{4}$.

In our case first nasopharyngeal aspirate was negative, but deep tracheal aspirate was positive after 2 days. More data is warranted to ensure whether deep tracheal aspirate is needed to diagnose COVID-19 sensitively.

In our case although relation between Steven Johnson Syndrome and COVID-19 is not clear, as amoxicilin clavulonate could also be cause of the syndrome. But the possibility of presentation of COVID-19 as rash or even Steven Johnson Syndrome should be kept in mind in cases that are presenting with pulmonary findings, similar to our case.

References

1. Dong Y, Mo X, Hu Y, et al. Epidemiological Characteristics of 2143 Pediatric Patients With 2019 Coronavirus Disease in China [published online ahead of print, 2020 Mar 16]. Pediatrics . 2020;e20200702. doi:10.1542/peds.2020-0702

2. Lu X, Zhang L, Du H, et al. SARS-CoV-2 Infection in Children [published online ahead of print, 2020 Mar 18]. N Engl J Med . 2020;10.1056/NEJMc2005073. doi:10.1056/NEJMc2005073

3. Fang F, Luo XP. Zhonghua Er Ke Za Zhi . 2020;58 (2):81-85. doi:10.3760/cma.j.issn.05781310.2020.02.001

4. de Wilde AH, Zevenhoven-Dobbe JC, van der Meer Y, et al. Cyclosporin A inhibits the replication of diverse coronaviruses. J Gen Virol . 2011;92 (Pt 11):2542-2548. doi:10.1099/vir.0.034983-0 


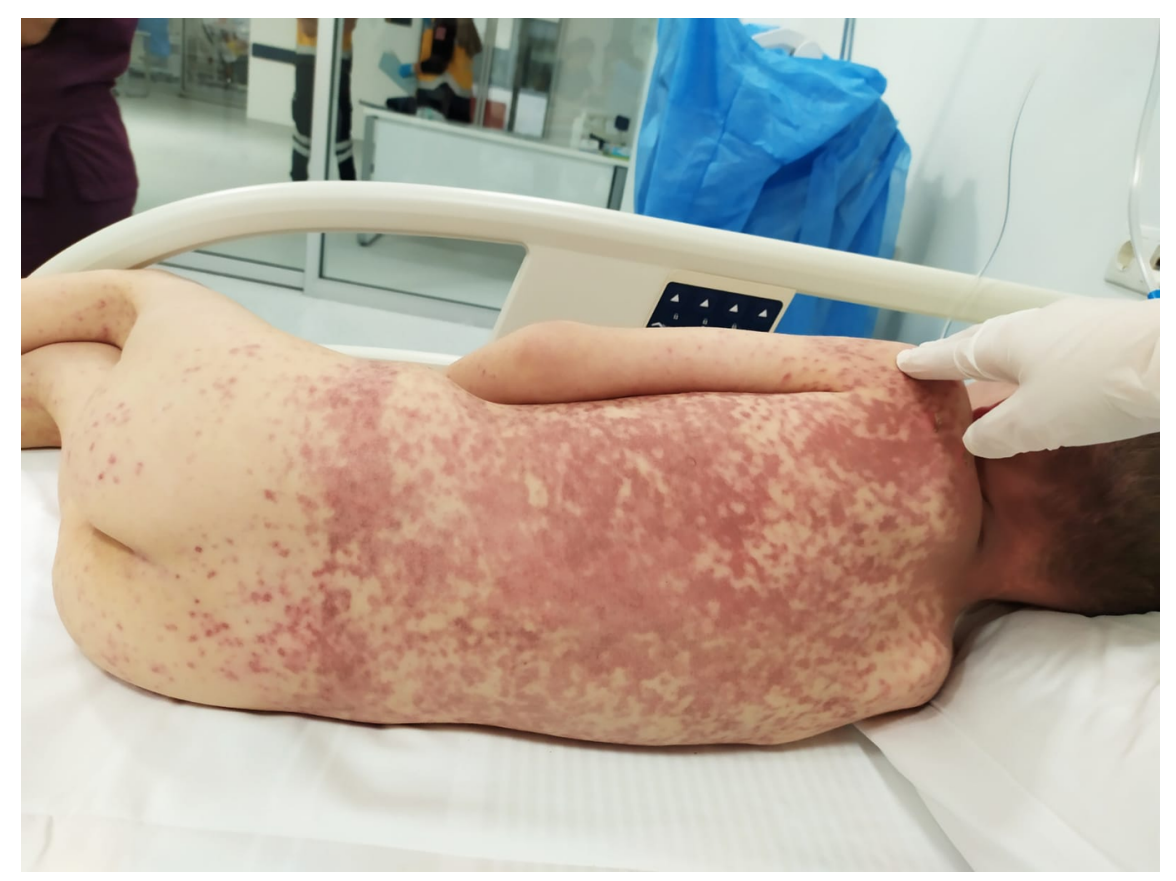

Figure1.a\&b. Trunk lesions of the patient

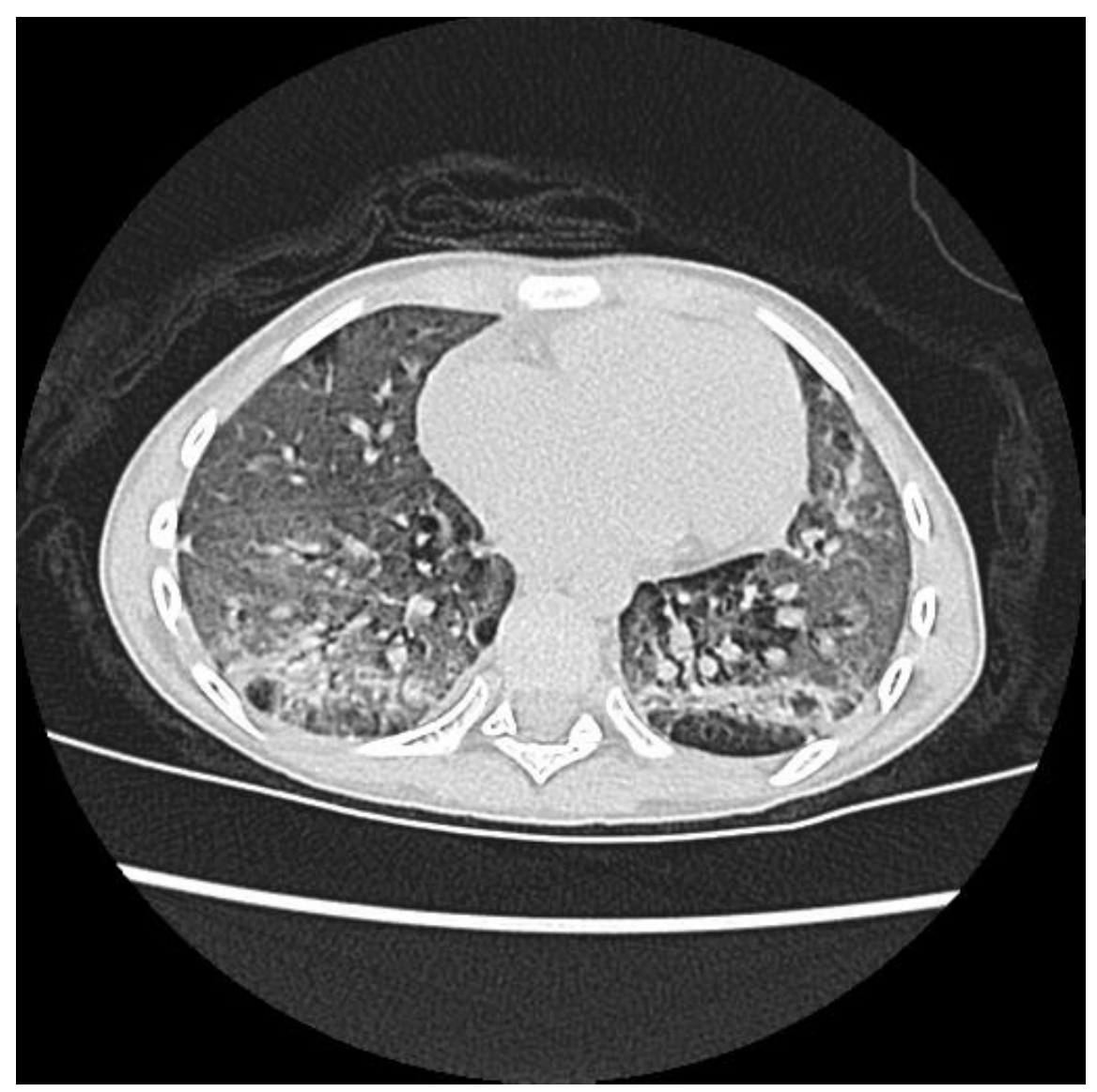




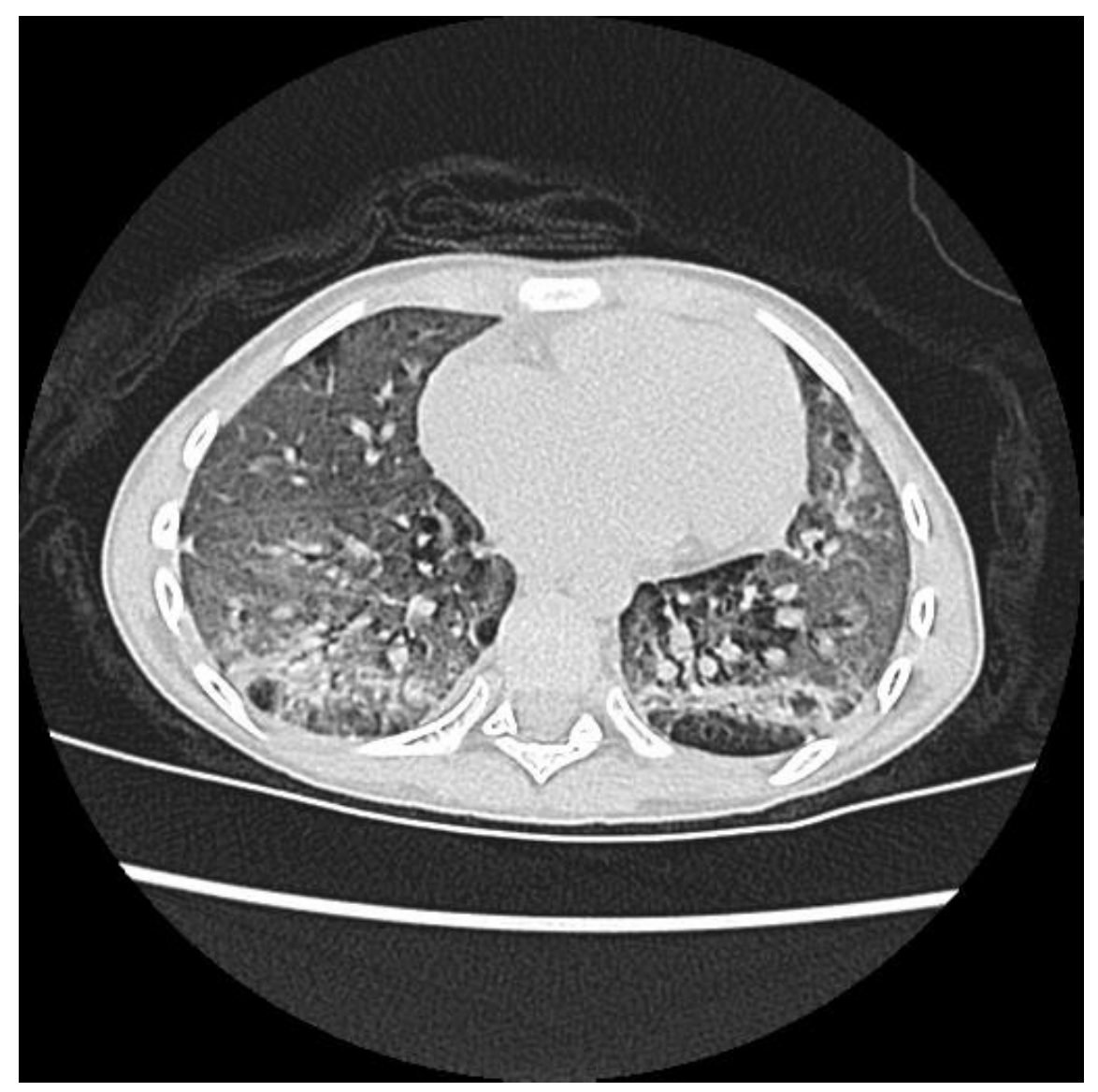

Figure 2.a\&b. Computed tomography images of the patient 